\title{
Expression of AEG-1 in human T-cell lymphoma enhances the risk of progression
}

\author{
JIAQIN YAN, MINGZHI ZHANG, QINGJIANG CHEN and XUDONG ZHANG
}

Department of Oncology, The First Affiliated Hospital, Zhengzhou University, Zhengzhou, Henan 450052, P.R. China

Received June 5, 2012; Accepted August 22, 2012

DOI: $10.3892 /$ or.2012.2055

\begin{abstract}
The aim of this study was to examine the expression and role of astrocyte elevated gene-1 (AEG-1) in biological processes of T-cell non-Hodgkin's lymphoma (T-NHL). AEG-1 expression in T-NHL patients was characterized with immunohistochemistry. The expression of AEG-1, survivin, Bcl-2 and Bax in Jurkat and Hut-78 cells was detected by real-time PCR and western blotting. Cell proliferation, cell cycle and apoptosis were measured by MTT and flow cytometry. MMP-2/-9 activity was detected by gelatin zymography. Of the studied tumors, 104 (80.62\%) exhibited cytoplasmic AEG-1 immunostaining. AEG-1-siRNA in Jurkat and Hut-78 cells suppressed cell proliferation and induced cell apoptosis, inhibited survivin and Bcl-2/Bax protein expression as well as MMP-2/-9 activity. Downregulation of AEG-1 using siRNA could provide a potential approach for gene therapy against T-NHL, and the antitumor effects may be associated with inhibition of survivin and Bcl-2/ Bax protein expression and MMP-2/-9 activity.
\end{abstract}

\section{Introduction}

Malignant lymphomas (MLs) are the lymph node and/or extranodal lymphoid malignancies. The lymphoma, in histopathology, is broadly divided into Hodgkin's lymphoma (HD) and nonHodgkin's lymphoma (NHL). Within both subsets are numerous variations with distinct biological, molecular, and cytogenetic characteristics (1). NHL is the most common malignant tumor with rapid incidence growth, a high mortality rate in children and adolescents and a serious harm to human health (2). One type of NHL is T-cell lymphoma (T-NHL). In recent years, with the rapid development of molecular biology and the completion of the Human Genome Project indicating that all tumors are the

Correspondence to: Dr Mingzhi Zhang, Department of Oncology, The First Affiliated Hospital, Zhengzhou University, 1 Jianshe East Road, Zhengzhou, Henan 450052, P.R. China

E-mail: mingzhi_zhang68@126.com

Abbreviations: AEG-1, astrocyte elevated gene-1; T-NHL, T-cell non-Hodgkin's lymphoma

Key words: T-cell non-Hodgkin's lymphoma, astrocyte elevated gene-1, Jurkat, Hut-78, RNA interference, apoptosis, gene therapy result of abnormal gene expressions, the causes of lymphoma are now better understood (3). Studies have shown that abnormal gene expression caused by oncogenes (4) and tumor suppressor gene mutations (5), chromosomal translocations (6) and viral infections are also significant causes of T-NHL (7).

Astrocyte elevated gene-1 (AEG-1) was originally characterized as a human immunodeficiency virus (HIV)-1-inducible gene in primary human fetal astrocytes (8). The AEG-1 gene is located on 8q22.1, there are 3611 pairs of bases, including 11 introns and 12 exons, encoding 582 amino acids. Its expression product MTDH distributed in the perinuclear cytoplasm is a 64-kDa transmembrane protein (9). In the study of molecular signaling pathways, AEG-1 is a transmembrane protein in the endoplasmic reticulum, nuclear membrane and nucleolus (10). In previous in-depth studies of AEG-1, it was found to promote the occurrence of neurodegeneration, as well as tumor progression and metastasis. AEG-1 expression is markedly induced by oncogenic Ha-ras, activating the phosphatidylinositol 3-kinase signaling pathway that augments binding of c-Myc to key E-box elements in the AEG-1 promoter, thereby regulating AEG-1 transcription (11). AEG-1 expression is elevated in diverse neoplastic states, it cooperates with Ha-ras and c-myc to promote transformation, and its overexpression augments invasion of transformed cells. These results strongly suggest that AEG-1 may play a functionally critical role in Ha-ras-mediated tumorigenesis; therefore, the role of AEG-1 in tumor growth, angiogenesis, and metastasis is studied extensively (12-14).

In the present study, we used immunohistochemistry to detect AEG-1 expression in T-NHL, and RNA interference (RNAi) technology to silence the expression of AEG-1 in the human T-NHL cell lines Hut 78 (cutaneous T-cell lymphoma) and Jurkat (adult T-cell leukemia/lymphoma). In vitro experiments described herein demonstrate that AEG-1 abnormal expression in T-NHL and the capability of tumor growth and metastasis is reduced, whereas apoptosis is induced in AEG-1siRNA transfected Jurkat and Hut-78 cells. In addition, we demonstrate for the first time the molecular mechanisms of the antitumor effects of AEG-1 knockdown, which could lay a foundation for genetic therapy for T-NHL.

\section{Materials and methods}

Cell lines. The T-NHL cell lines Jurkat and Hut-78 were used in these experiments. Jurkat and Hut-78 were maintained in the Key Clinical Laboratory of Henan Province. Cells were 
cultured in RPMI-1640 medium supplemented with $10 \%$ heatinactivated FBS (fetal bovine serum), $50 \mathrm{U} / \mathrm{ml}$ penicillin and $50 \mathrm{U} / \mathrm{ml}$ streptomycin (Sigma-Aldrich, St. Louis, MO, USA) at $37^{\circ} \mathrm{C}$ in a humidified atmosphere containing $5 \% \mathrm{CO}_{2}$.

Clinical samples. From 2008 to 2011, 129 patients who were diagnosed with T-NHL and received standard chemotherapy after lymph node biopsy at The First Affiliated Hospital of Zhengzhou University were included in the study after obtaining their oral and written informed consent. The biopsy specimen of each patient was prepared by the Department of Clinical Pathology for paraffin-embedded tumor tissue sections. Information on histopathological diagnosis was extracted from medical records and reviewed by a specialist in gynecologic tumor pathology. The control group consisted of 17 samples of lymph node that were obtained from standard operations. This study was reviewed and approved by the ethics committee of the medical faculty at the First Affiliated Hospital of Zhengzhou University.

Antibodies and reagents. Antibodies against AEG-1, survivin, Bcl-2 and Bax were purchased from Santa Cruz Biotechnology (Santa Cruz, CA, USA). $\beta$-actin antibody and secondary goat anti-rabbit and goat anti-mouse alkaline phosphatase antibodies were also purchased from Santa Cruz Biotechnology. All antibodies were used for western blot analysis at a dilution of 1:2,000 and 1:5,000, respectively. RPMI-1640, FBS, Lipofectamine $^{\mathrm{TM}} 2000$ and TRIzol reagent were purchased from Invitrogen Co. (Carlsbad, CA, USA). MTT and DMSO were obtained from Sigma.

Immunohistochemistry. Standard immunoperoxidase procedures were used to visualize AEG-1 expression. Briefly, paraffin sections were deparaffinized in xylene, followed by a graded series of alcohols (100, 95 and $75 \%$ ) and re-hydrated in water followed by Tris-buffered saline. Following antigen retrieval, slides were incubated with $3 \% \mathrm{H}_{2} \mathrm{O}_{2}$ to inhibit endogenous peroxidase. Slides were then blocked with 5\% normal serum and incubated with anti-AEG-1 antibody. After washing, the tissue sections were treated with biotinylated antirabbit secondary antibody (Zymed Laboratories Inc., South San Francisco, CA, USA), followed by further incubation with streptavidin-horseradish peroxidase complex (Zymed). Tissue sections were then immersed in 3,3'-diaminobenzidine and counterstained with $10 \%$ Mayer's hematoxylin, dehydrated and mounted.

AEG-1 expression in paraffin-embedded sections was viewed and scored separately by two independent pathologists, who were blinded to the histopathological features and patient data, and AEG-1 expression was determined by combining the proportion of positively stained tumor cells and the intensity of staining (15).

MTT cell viability assay. To quantify cell proliferation, Jurkat and Hut-78 cells and stably transfected cells (Jurkat-RNAi and Hut-78-RNAi) were seeded in a 96-well plate at a concentration of $2.5 \times 10^{4} / \mathrm{ml}(100 \mu \mathrm{l} / \mathrm{well})$. Six parallel wells were assigned to each group. Then, $20 \mu \mathrm{l} / \mathrm{well}$ of $5 \mathrm{mg} / \mathrm{ml}$ MTT (3-(4,5-dimethylthiazol-2-yl)-2,5-diphenyltetrazolium bromide) was added at $1,2,3,4,5$ and 6 days after seeding and were then incubated for another $4 \mathrm{~h}$. The supernatant was removed and the product converted from MTT was dissolved by adding $150 \mu 1 /$ well dimethylsulfoxide (DMSO). The plate was gently shaken for $15 \mathrm{~min}$ at room temperature and an enzyme-linked immunosorbent assay reader was used to measure the absorbance of each well at $570 \mathrm{~nm}$. The growth curve was drawn according to the cell growth rate.

RNA extraction, reverse transcription and real-time PCR. Total-RNA from cultured cells was extracted using the TRIzol reagent according to the manufacturer's instructions. The cDNA synthesis was carried out according to the protocol of the Takara Reverse Transcription System for real-time PCR [Takara Biotechnology (Dalian) Co., Ltd., China] with a starting amount of $2 \mu \mathrm{g}$ RNA and reverse transcription performed with random primers. Real-time PCR primers were designed according to www.ncbi.nlm.nih.gov. Primer sequences are: AEG-1 forward, 5'-CTCGGGCTGCTGCTGCTGTT-3', and reverse, 5'-CAGC AAGGCCAGGTCGTCGG-3'; Bax forward, 5'-GGCCGG GTTGTCGCCCTTTT-3', and reverse, 5'-CCGCTCCCGGA GGAAGTCCA-3'; Bcl-2 forward, 5'-GGACGGGGTGA ACTGGGGGA-3', and reverse, 5'-GGTGTGCAGGTGCCGG TTCA-3'; survivin forward, 5'-CGCTTTGCCACGGTGGT GGA-3', and reverse, 5'-CAGGGGCGACATCTCCC GGT-3'; $\beta$-actin forward, 5'-CGACAACGGCTCCGGCATGT-3', and reverse, 5'-TGGGCCTCGTCGCCCACATA-3'. Real-time PCR analysis was carried out on a LightCycler real-time PCR instrument using SYBR Green I kit (Tiangen Biotech Co., Ltd., Beijing, China) according to the manufacturer's instructions. Each reaction was performed in triplicate. Data were analyzed with the Sequence detector software (v1.9, Applied Biosystems) and analyzed using the $2^{-\Delta \Delta C T}$ method as previously described (16).

Western blot analysis. Total proteins were extracted by lysing cells in buffer containing $50 \mathrm{mM}$ Tris $\mathrm{pH} 7.4,150 \mathrm{mM} \mathrm{NaCl}$, $0.5 \% \mathrm{NP}-40,50 \mathrm{mM} \mathrm{NaF}, 1 \mathrm{mM} \mathrm{Na} \mathrm{VO}_{4}, 1 \mathrm{mM}$ phenylmethylsulfonyl fluoride, $25 \mathrm{mg} / \mathrm{ml}$ leupeptin and $25 \mathrm{mg} / \mathrm{ml}$ aprotinin. The lysates were cleared by centrifugation and the supernatants were collected. Proteins were extracted using the protein extraction kit according to the manufacturer's instructions. Protein concentration was determined using protein assay reagent (Bio-Rad, Hercules, CA, USA). Equal amounts of protein were separated on SDS-PAGE, transferred to PVDF membranes, incubated with antibodies against AEG-1, survivin, Bcl-2 and Bax, $\beta$-actin, followed by incubation with the secondary antibodies. The membrane was then washed three times with TBST and visualized with diaminobenzidine. Quantification of the proteins was detected with the ECL system (Pierce Biotechnology Inc., Rockford, IL, USA). Each value represents the mean of triple experiments, and is presented as the relative density of protein bands normalized to $\beta$-actin.

Plasmid vector constructs, transfection. The plasmid constructs carrying siRNA (pSUPER-EGFP-AEG-1) against AEG-1 were designed and constructed as previously described (17). The following siRNA sequences were cloned into the pSUPEREGFP plasmid: forward, 5'-GCTGATCCCAACTCTGATTTT CAAGAGAAATCAGAGTTGGGATCAGC-3', and reverse, 
A

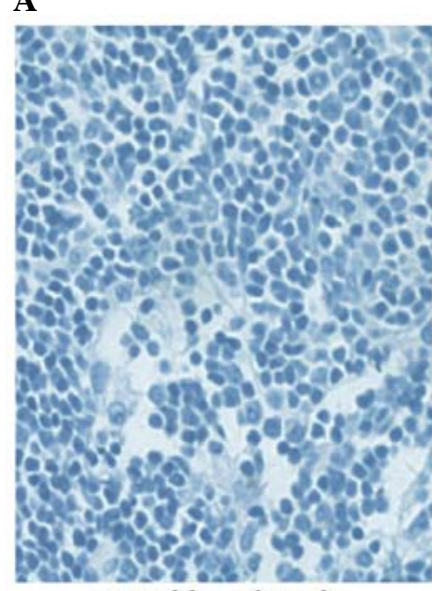

normal lymph node
B

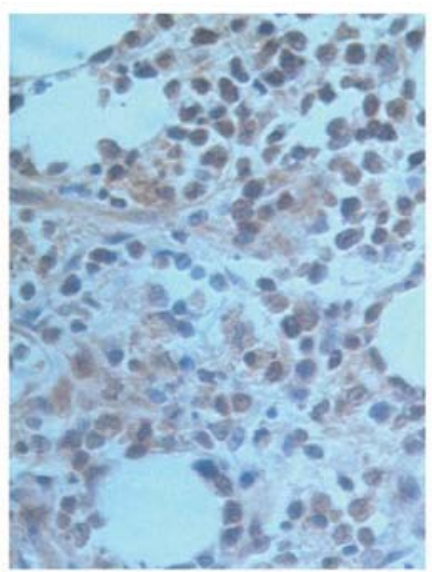

T-cell lymphoma
C

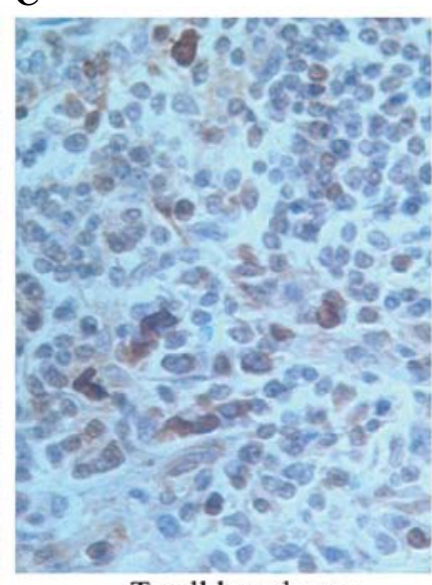

T-cell lymphoma

Figure 1. AEG-1 protein is overexpressed in T-NHL tissue. (A) AEG-1 expression in cells of normal lymph node tissue was not detectable (x400). (B and C) AEG-1 expression was positive in the cells of primary lesions of T-NHL (x400).

\section{5'-CGACTAGGGTTGAGACTAAAAGTTCTCTTTAGTCT} CAACCCTAGTCG-3'. The recombinant plasmids were transformed into competence bacillus coli and cultured; they were then extracted, purified, identified, measured and stored at $-20^{\circ} \mathrm{C}$. The T-NHL cell line Jurkat and Hut-78 cells were transfected with pSUPER-EGFP-AEG-1 using Lipofectamine 2000 (Invitrogen) according to the manufacturer's instructions. Forty-eight hours after transfection, Jurkat and Hut-78 cells were diluted and selected in the medium containing $500 \mu \mathrm{g} / \mathrm{ml} \mathrm{G} 418$ for 4 weeks. Then, the positive clones were picked up and expanded to establish cell lines after maintaining to select for 4 weeks. The stable transfection cell clones Jurkat-RNAi and Hut-78-RNAi were verified for RT-PCR and western blot analysis.

Cell cycle analysis. Flow cytometric analysis of PI-stained cells was performed to demonstrate the cell cycle of the different cell groups. Each different group of cells was cultured in a $10 \mathrm{~cm}$ dish, respectively. After culturing for $48 \mathrm{~h}$, the cells were harvested, washed and fixed in $70 \%$ ethanol for $30 \mathrm{~min}$. After fixation, cells were resuspended in $1 \mathrm{ml}$ propidium iodide (PI) staining buffer (0.1\% Triton X-100, $100 \mathrm{mg} \mathrm{ml}-1$ RNase A, $500 \mathrm{mg} \mathrm{ml}-1 \mathrm{PI}$ in PBS) at $37^{\circ} \mathrm{C}$ for $30 \mathrm{~min}$ and the proportion of cells in a particular phase of the cell cycle was determined by flow cytometry.

Annexin V-FITC flow cytometric analysis. Annexin V-FITC apoptosis detection kit (BD Biosciences, San Jose, CA, USA) was used to detect early apoptosis. In brief, after culturing for $48 \mathrm{~h}$, each group of cells was harvested, washed twice with pre-chilled PBS and resuspended in binding buffer (HEPES$\mathrm{NaOH} 10 \mathrm{mM}$ pH 7.4, $144 \mathrm{mM} \mathrm{NaCl}$ and $25 \mathrm{mM} \mathrm{CaCl}_{2}$ ) at a concentration of $1 \times 10^{6}$ cells $/ \mathrm{ml}$. One hundred microliters of this solution ( $1 \times 10^{5}$ cells) was mixed with $5 \mu 1$ of Annexin V-FITC and $5 \mu \mathrm{l}$ of PI (BD Biosciences) according to the manufacturer's instructions. The mixed solution was gently vortexed and incubated in the dark at room temperature $\left(25^{\circ} \mathrm{C}\right)$ for $15 \mathrm{~min}$. Four hundred microliters of $1 \mathrm{X}$ dilution buffer were added to each tube and cell apoptosis analysis was performed by flow cytometry (BD FACSCalibur) within
$1 \mathrm{~h}$. At least 10,000 events were recorded and represented as dot plots. Cells that stained positive for Annexin V-FITC and negative for PI were counted as apoptotic cells.

Analysis of MMP-2 and MMP-9 activities by gelatin zymography. The activities of MMP-2 and MMP-9 tumor cells were assayed by gelatin zymography. Briefly, each group of cells was incubated with serum-free medium for $24 \mathrm{~h}$. The conditioned medium was then harvested and concentrated by ultra-filtration centrifugation. The sample $(20 \mu \mathrm{g})$ was mixed with loading buffer and subjected to gelatin zymogram gel (10\% SDS-polyacrylamide gel, $0.1 \%$ gelatin). Electrophoresis was performed at $100 \mathrm{~V}$ for $3 \mathrm{~h}$ at $4^{\circ} \mathrm{C}$. Following electrophoresis, gels were washed with washing buffer $(2.5 \%$ Triton $\mathrm{X}-100$ in $\mathrm{ddH}_{2} \mathrm{O}$ ) at room temperature to remove SDS, followed by incubation at $37^{\circ} \mathrm{C}$ in reaction buffer $(40 \mathrm{mM}$ Tris- $\mathrm{HCl}$, $\mathrm{pH} 8.0,10 \mathrm{mM} \mathrm{CaCl}_{2}, 0.02 \% \mathrm{NaN}_{3}$ ). After $16 \mathrm{~h}$, the gels were stained with Coomassie blue R-250 (0.125\% Coomassie blue R-250, 50\% methanol, $10 \%$ acetic acid) for $3 \mathrm{~h}$ and destained with destaining solution (20\% methanol, $10 \%$ acetic acid, $70 \% \mathrm{ddH}_{2} \mathrm{O}$ ) until the clear bands were visualized. MMP-2 and MMP-9 (gelatinase) activity was visible as clear bands against the dark blue background, indicating proteolysis of the substrate protein, gelatin.

Statistical analysis. Each experiment was performed at least twice; results are expressed as mean \pm SEM using Excel software (Microsoft, Redmond, WA, USA) for calculation. The SPSS13.0 software package (SPSS, Inc., Chicago, IL, USA) was used for all statistical analyses. Statistical analysis was performed by ANOVA. Student's t-test was also performed and the results were considered statistically significant at $\mathrm{P}<0.05$.

\section{Results}

Upregulation of AEG-1 in T-NHL. To examine whether the AEG-1 protein is upregulated in T-NHL tissues, 129 paraffinembedded, archived T-NHL tissues were examined by immunohistochemical staining with an antibody against human AEG-1. As shown in Fig. 1, the AEG-1 protein was 

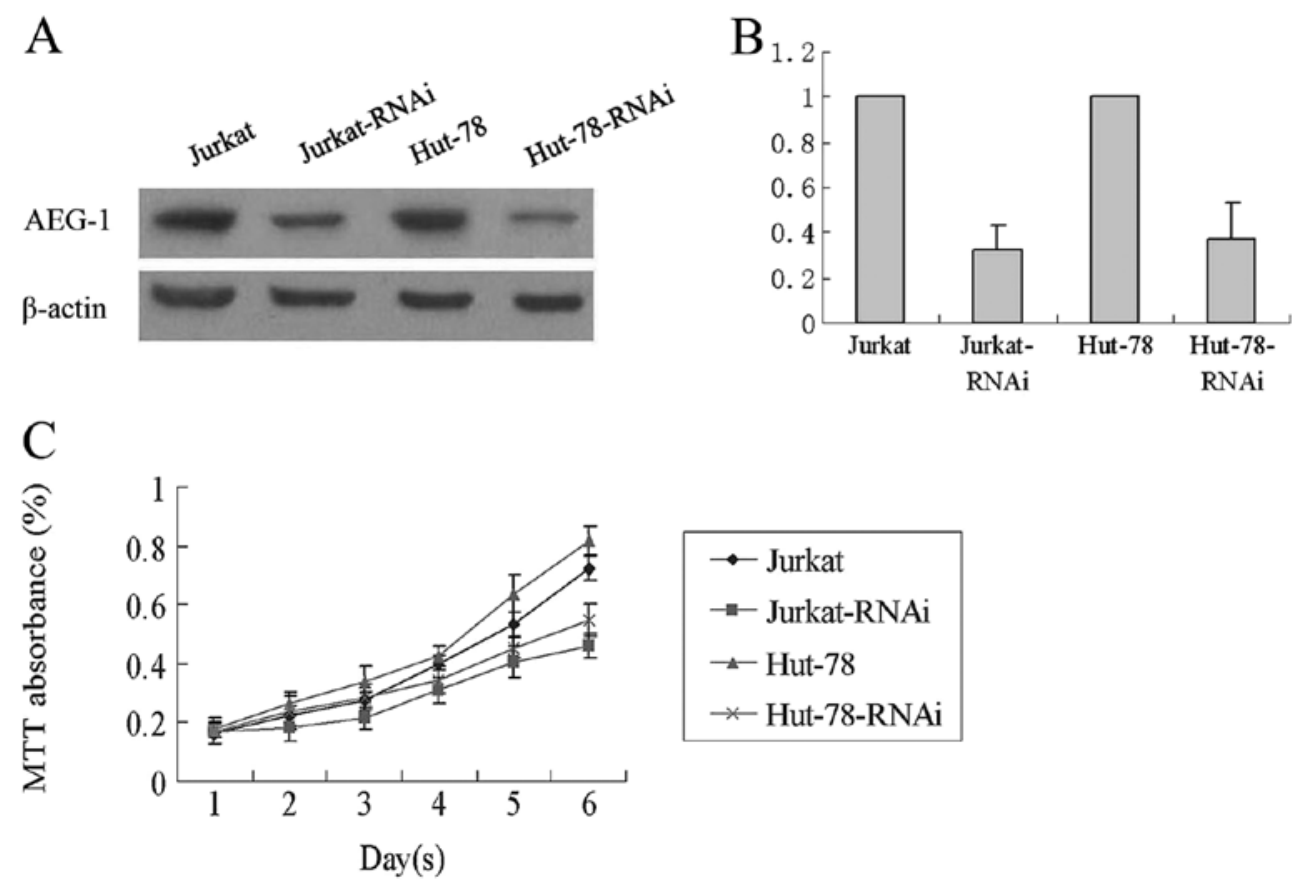

Figure 2. Knockdown of endogenous AEG-1 inhibits cell growth. (A and B) AEG-1 expression in Jurkat and Hut-78 cells analyzed by western blotting and RT-PCR. $\beta$-actin was used as a loading control. (C) Silencing endogenous AEG-1 inhibits Jurkat and Hut-78 cell growth as determined by MTT assays.

found to be upregulated in T-NHL compared with normal lymph node tissues. High levels of AEG-1 expression were present in areas containing tumor cells of the T-NHL. By contrast, AEG-1 was barely detectable in the normal lymph node tissues $(\mathrm{P}<0.01$, Fig. 1$)$.

AEG-1-siRNA inhibits Jurkat and Hut-78 cell proliferation. Previous studies have shown that the expression of AEG-1 is associated with T-NHL growth. To further investigate the biological role of AEG-1 expression in T-NHL progression, the T-NHL cell lines Jurkat and Hut-78 were established to stably low-expressed AEG-1. Western blotting and RT-PCR assay results indicated that AEG-1-infected Jurkat-RNAi and Hut-78-RNAi cells have low AEG-1 mRNA and protein expression ( $\mathrm{P}<0.01$, Fig. 2A and B). The effect of AEG-1siRNA on T-NHL cell proliferation was determined by MTT assay. As shown in Fig. 2, cell proliferation was remarkably inhibited in the Jurkat-RNAi and Hut-78-RNAi groups, when compared with the Jurkat and Hut-78 groups ( $\mathrm{P}<0.05$, Fig. 2C).

AEG-1-siRNA arrests Jurkat and Hut-78 cells at the G0/G1 phase. To analyze the mechanisms by which AEG-1-siRNA inhibits cell proliferation, the cell cycle of Jurkat and Hut-78 cells as well as transfected Jurkat-RNAi and Hut-78-RNAi cells were analyzed by flow cytometry. As shown in Fig. 3, the percentage of Jurkat and Hut-78 cells at the G0/G1 phase was increased from 54.95 and 48.52 to 69.52 and $67.19 \%$; the S-phase cells were decreased from 32.63 and 36.4 to 21.36 and $24.44 \%$ ( $\mathrm{P}<0.05$, Fig. 3). These data show that silencing of AEG-1 can arrest cells at the G0/G1 phase.

AEG-1-siRNA promotes Jurkat and Hut-78 cell apoptosis. To further evaluate whether knockdown of AEG-1 induces Jurkat and Hut-78 cell apoptosis, at $48 \mathrm{~h}$ after culture, the cells were harvested and analyzed by flow cytometry. As shown in Fig. 4, the apoptosis rates of the Jurkat-RNAi and Hut-78-RNAi groups were 13.2 and $11.51 \%$, respectively, and were $>1.64 \%$ in the Jurkat and $1.51 \%$ in the Hut-78 group ( $\mathrm{P}<0.01$, Fig. 4).

Molecular mechanisms of the antitumor effects by AEG-1siRNA. Since deregulation of the AEG-1 expression appears tightly linked to the apoptosis of Jurkat and Hut-78 cells, we further investigated whether survivin, Bcl-2 and Bax could be regulated by AEG-1. Western blot analysis revealed that ectopic expression of AEG-1 affects the expression of survivin, $\mathrm{Bcl}-2$ and $\mathrm{Bax}$, whereas the expression of Bax is upregulated and survivin and $\mathrm{Bcl}-2$ is downregulated in Jurkat-RNAi and Hut-78-RNAi cells compared with control cells $(\mathrm{P}<0.01$, Fig. 5A). Furthermore, real-time PCR results indicated that the modulation of survivin, Bcl-2 and Bax by AEG-1 were regulated at the transcriptional level ( $\mathrm{P}<0.01$, Fig. 5B). These data demonstrate that knockdown of AEG-1 may affect survivin, Bcl-2 and Bax expression, which all play an important role in tumor progression.

AEG-1-siRNA arrests MMP-2 and MMP-9 activities in Jurkat and Hut-78 cells. Since MMP-2 and MMP-9 play a critical role in tumor cell invasion, we examined the effects of AEG-1 silencing on the enzyme activities of MMP-2 and MMP-9 using gelatin zymography. Each group of cells was incubated in serum-free medium. After culturing for $24 \mathrm{~h}$, conditioned media in each group were collected and the expression levels of interest were detected using zymography. The gelatinolytic activities of both MMP-2 and MMP-9 were found to be markedly reduced in the Jurkat-RNAi and Hut-78-RNAi groups compared with the control groups $(\mathrm{P}<0.01$, Fig. 6). There was a significant difference between the groups $(\mathrm{P}<0.05)$. 
A
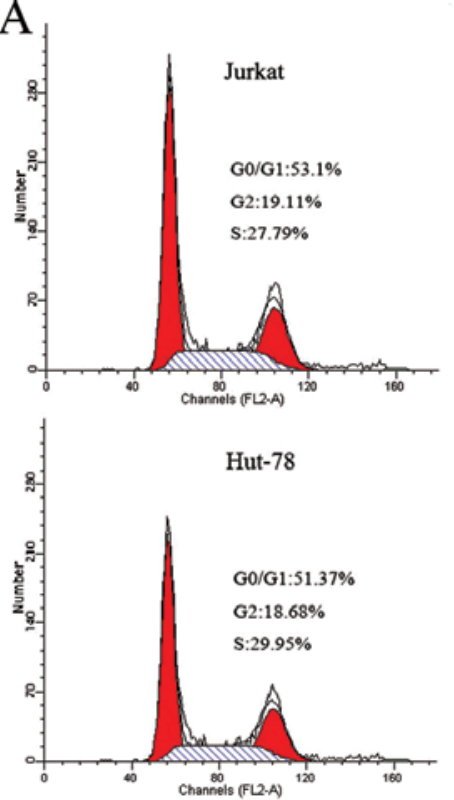
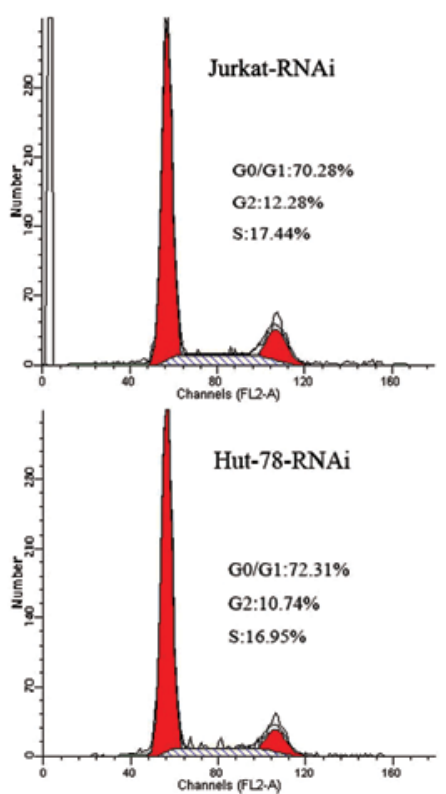

B

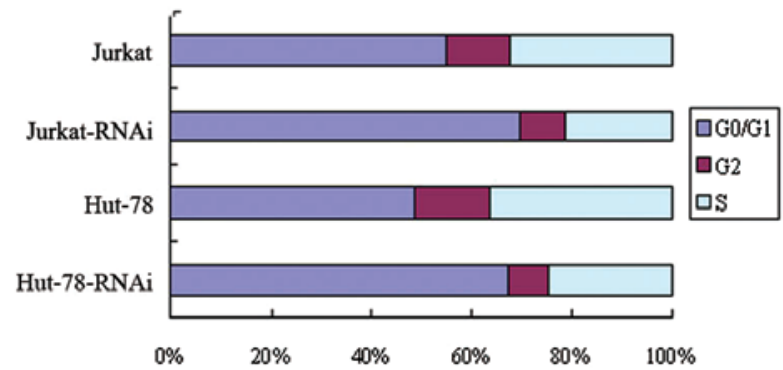

Figure 3. Knockdown of endogenous AEG-1 arrests Jurkat and Hut-78 cells at the G0/G1 phase. (A) Flow cytometry measurements of cell-cycle distribution of Jurkat and Hut-78 cells. (B) Plots showing distribution of Jurkat and Hut-78 cells in sub-G1, G0/G1 and G2/M phase compartments.

A
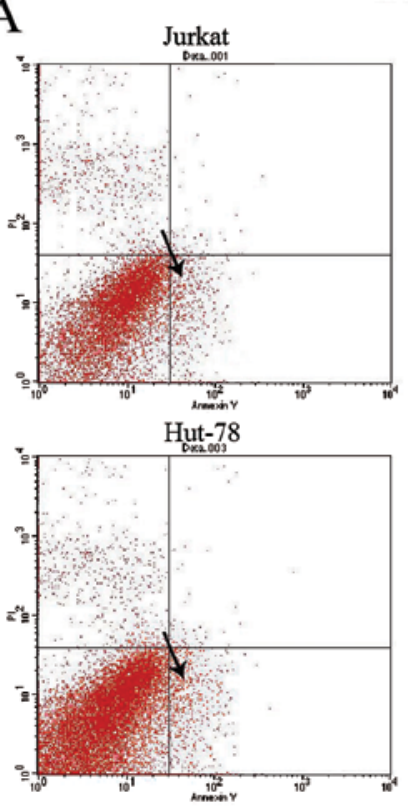
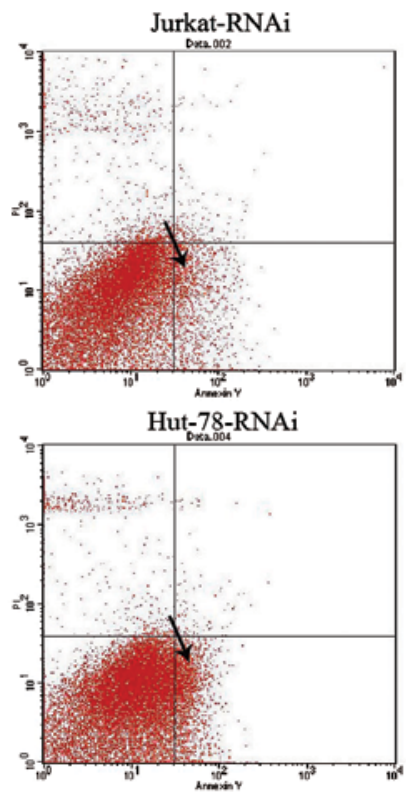

$\mathrm{B}$

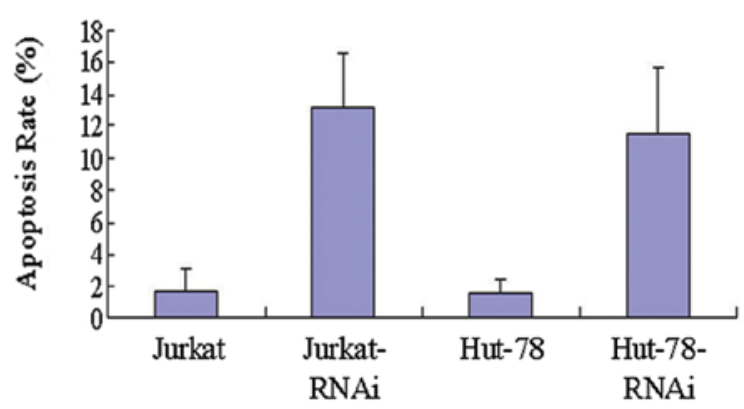

Figure 4. Knockdown of endogenous AEG-1 induced apoptosis of Jurkat and Hut-78 cells. (A) Flow cytometry measurements of the cell apoptosis rate of Jurkat and Hut-78 cells. (B) Histogram comparing the percentage of apoptotic cells in each group.
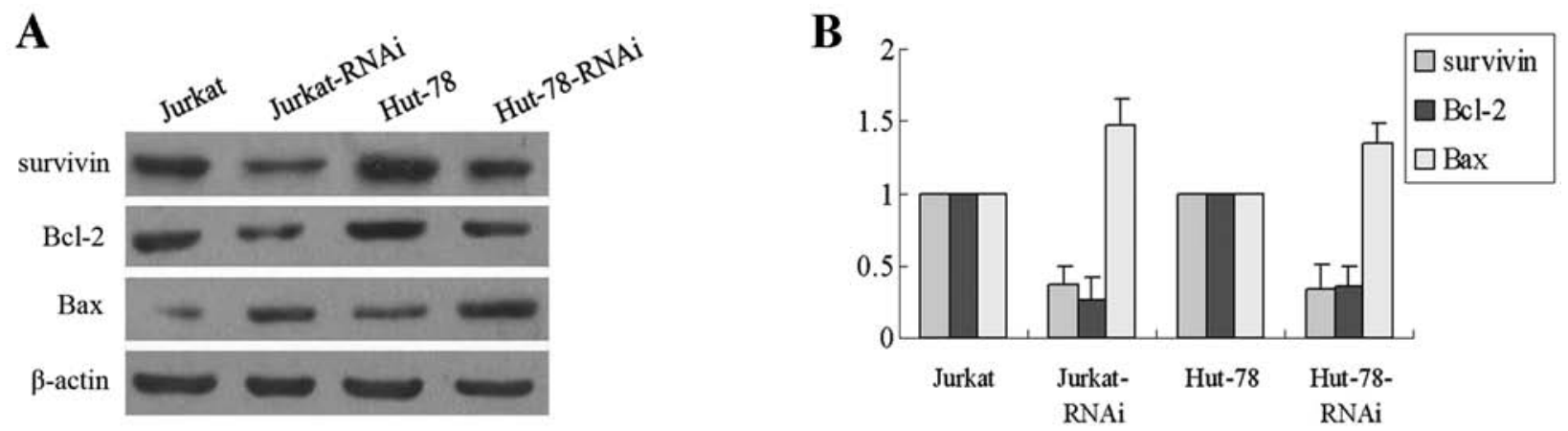

Figure 5. The effect of knockdown of endogenous AEG-1 on the expression of survivin, Bcl-2 and Bax in T-NHL cells in vitro. (A) Western blot analysis of the expression of the survivin, Bcl-2 and Bax proteins in each group. (B) Relative mRNA analysis of the expression of survivin, Bcl-2 and Bax mRNAs in each group. 

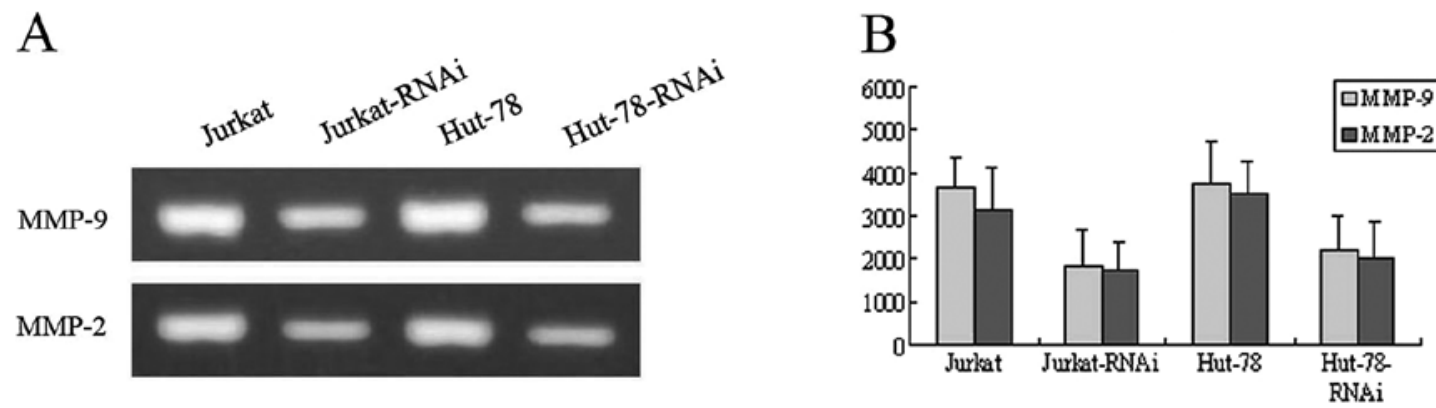

Figure 6. Gelatin zymography analysis of MMP-2 and MMP-9 activities in the different groups. Cells were cultured for $24 \mathrm{~h}$ and conditioned media were used for the measurement of MMP-2 and MMP-9 activities by gelatin zymography. (A) Images of the MMP-2 and MMP-9 bands, which are representative of three independent experiments, are shown. (B) Quantitative analysis of the bands.

\section{Discussion}

T-NHL is a group of malignant tumors with high heterogeneity from the lymphatic system accounting for $10-15 \%$ of all NHL, with incidence rates increasing year by year (18). Despite advances in the medical treatments, and due to its intrinsic properties, T-NHL has a poorer prognosis compared to its B-cell counterparts $(19,20)$. T-NHL progression includes tumor cell proliferation, migration and invasion. Therefore, effectively inhibiting the proliferative and metastatic biological behavior of T-NHL cells is a key problem to improving the outcome (21-24).

Recent studies have indicated that AEG-1 plays an important role in the biological processes of many types of cancer, and the incidences of tumor development, invasion, metastasis and resistance are closely related (25-28). In human gallbladder carcinoma (GBC), there is increased AEG-1 expression in GBC samples and highly invasive GBC-SD cell lines at both the protein and mRNA levels, and this is strongly correlated with differentiation degree, Nevin stage, Ki-67 expression and survival time (29). AEG-1 is overexpressed in a great portion of epithelial ovarian cancer (EOC) patients with peritoneal dissemination and/or lymph node metastasis and this may be clinically useful for predicting metastasis in EOC (30). AEG-1 overexpression was also positively correlated with the International Federation of Gynecology and Obstetrics stage, depth of myometrial invasion, lymph node metastasis, lymph vascular space invasion, and recurrence. Patients with high AEG-1 expression had a significantly poor overall and disease-free survival (31). In this study, we used immunohistochemical analysis to show that AEG-1 is highly expressed in human T-NHL and it is confined in the endochylema of the tumor cells, suggesting that this protein is significantly involved in T-NHL growth.

Although AEG-1 does not affect MDR1 gene transcription, it facilitates association of MDR1 mRNA to polysomes, resulting in increased translation, and AEG-1 also inhibits ubiquitination and subsequent proteasome-mediated degradation of the MDR1 protein. This suggests that inhibition of AEG-1 might provide a means of more effectively using chemotherapy to treat human hepatocellular carcinoma (HCC). Inhibition of AEG-1 expression by short hairpin RNA can increase doxorubicin toxicity of chemotherapy drugs to aggressive human HCC cells compared with either agent alone (32). Inhibition of AEG-1 expression also clearly inhibited SGC-7901 cell growth and enhanced cell apoptosis by reducing phosphorylation of AKT and glycogen synthase kinase (GSK)-3 $\beta$ (Ser 9) and decreasing the level of $\beta$-catenin, lymphoid enhancer binding factor 1 (LEF1), and cyclin D1 (33). These reports demonstrate that silencing the expression of AEG-1 in cancer affects the different types of tumor cell proliferation, apoptosis, cell cycle, chemoresistance, invasion and metastasis, but the effect of AEG-1-siRNA on T-NHL has yet to be explored. In this study, we showed that chemically synthesized siRNAs specifically targeting AEG-1 successfully knocked down the expression of AEG-1 in both protein and mRNA levels by 64-68\% in human T-NHL Jurkat and Hut-78 cells. The assays herein described detected the effects on the biological behavior of A549 cells in vitro. Using MTT, we were able to first show that the proliferation of the AEG-1-siRNA transfected T-NHL cells is significantly inhibited in vitro. Using flow cytometric analysis, we revealed that knockdown of AEG-1 expression induced apoptosis in Jurkat and Hut-78 cells. Based on these findings, we support that AEG-1 plays a key role in the proliferation of Jurkat and Hut-78 cells.

The mechanism of AEG-1 on tumor formation and development is associated with a wide range of downstream signaling pathways, which makes AEG-1 a potentially significant molecule and a complex network of signaling pathways in malignant progression of human tumors (34-36). However the molecular mechanism of apoptosis induced by the inhibition of AEG-1 expression remains unclear. In the current study, we established that downregulation of AEG-1 expression in Jurkat and Hut-78 cells suppressed the protein expression of survivin and Bcl-2, whereas it upregulated the protein expression of Bax, which are well known for mediating cell apoptosis (37-39). Therefore, this result explains, at least in part, why AEG-1-siRNA inhibited cell proliferation and induced apoptosis in Jurkat and Hut-78 cells.

At the molecular level, this study showed that upregulated AEG-1 in glioma cells interacted with MMP-9 promoter and transactivated MMP-9 expression, whereas knockdown of AEG-1 expression reduced the level of MMP-9 (40). Mechanistic studies conducted in vitro and in vivo revealed that AEG-1-mediated carcinogenesis and invasiveness might be through upregulation of MMP-2 (41). Our study also found the activities of MMP-2/-9 were also downregulated in AEG-1-siRNA transfected Jurkat and Hut-78 cells, and this may suggest that MMP-2/-9 are the downstream products of the AEG-1-induced cell signaling. MMPs are a family of enzymes that degrade proteins in tissue extracellular matrices, which are clearly involved in cancer progression, including 
tumor cell degradation of basement membranes and stroma and blood vessel penetration $(42,43)$. Consequently, the reduction of MMP-2/-9 activities by AEG-1-siRNA results in attenuating the metastatic potency of Jurkat and Hut-78 cells.

In conclusion, our findings clearly demonstrate that AEG-1 plays a crucial role in T-NHL growth and metastasis. Our study is the first to show that silencing of AEG-1 expression in Jurkat and Hut-78 cells by AEG-1-siRNA could inhibit cell growth and metastasis in vitro, and the antitumor effects may be associated with inhibition of survivin, the Bcl-2/Bax and MMP-2/-9 signal pathways in Jurkat and Hut-78 cells. Therefore, RNA interference targeting AEG-1 may be a beneficial potential tool for the gene therapy of T-NHL as well as other types of cancer at high levels of AEG-1 expression.

\section{References}

1. Ramsdale E, van Besien K and Smith SM: Personalized treatment of lymphoma: promise and reality. Semin Oncol 38: 225-235, 2011.

2. Hadzi-Pecova L, Petrusevska G and Stojanovic A: Non-Hodgkin's lymphomas: immunologic prognostic studies. Prilozi 28: 39-55, 2007.

3. Alizadeh AA, Ross DT, Perou CM and van de Rijn M: Towards a novel classification of human malignancies based on gene expression patterns. J Pathol 195: 41-52, 2001.

4. Huang Y, de Reyniès A, de Leval L, Ghazi B, Martin-Garcia N, Travert M, Bosq J, Brière J, Petit B, Thomas E, et al: Gene expression profiling identifies emerging oncogenic pathways operating in extranodal NK/T-cell lymphoma, nasal type. Blood 115: $1226-1237,2010$

5. Kleppe M, Tousseyn T, Geissinger E, Kalender Atak Z, Aerts S, Rosenwald A, Wlodarska I and Cools J: Mutation analysis of the tyrosine phosphatase PTPN2 in Hodgkin's lymphoma and T-cell non-Hodgkin's lymphoma. Haematologica 96: 1723-1727, 2011.

6. Fernberg P, Chang ET, Duvefelt K, Hjalgrim H, Eloranta S, Sørensen KM, Porwit A, Humphreys K, Melbye M and Ekström Smedby K: Genetic variation in chromosomal translocation breakpoint and immune function genes and risk of non-Hodgkin lymphoma. Cancer Causes Control 21: 759-769, 2010

7. Ramos JC and Lossos IS: Newly emerging therapies targeting viral-related lymphomas. Curr Oncol Rep 13: 416-426, 2011.

8. Emdad L, Sarkar D, Su ZZ, Randolph A, Boukerche H, Valerie K and Fisher PB: Activation of the nuclear factor kappaB pathway by astrocyte elevated gene-1: implications for tumor progression and metastasis. Cancer Res 66: 1509-1516, 2006.

9. Anttila V, Stefansson H, Kallela M, et al: Genome-wide association study of migraine implicates a common susceptibility variant on 8q22.1. Nat Genet 42: 869-873, 2010.

10. Kang DC, Su ZZ, Sarkar D, Emdad L, Volsky DJ and Fisher PB Cloning and characterization of HIV-1-inducible astrocyte elevated gene-1, AEG-1. Gene 353: 8-15, 2005.

11. Lee SG, Su ZZ, Emdad L, Sarkar D and Fisher PB: Astrocyte elevated gene-1 (AEG-1) is a target gene of oncogenic Ha-ras requiring phosphatidylinositol 3-kinase and c-Myc. Proc Natl Acad Sci USA 103: 17390-17395, 2006.

12. Emdad L, Sarkar D, Su ZZ, Lee SG, Kang DC, Bruce JN, Volsky DJ and Fisher PB: Astrocyte elevated gene-1: recent insights into a novel gene involved in tumor progression, metastasis and neurodegeneration. Pharmacol Ther 114: 155-170, 2007

13. Hu G, Wei Y and Kang Y: The multifaceted role of MTDH/AEG-1 in cancer progression. Clin Cancer Res 15: 5615-5620, 2009.

14. Yoo BK, Emdad L, Lee SG, Su ZZ, Santhekadur P, Chen D, Gredler R, Fisher PB and Sarkar D: Astrocyte elevated gene-1 (AEG-1): A multifunctional regulator of normal and abnormal physiology. Pharmacol Ther 130: 1-8, 2011.

15. Yu C, Chen K, Zheng H, Guo X, Jia W, Li M, Zeng M, Li J and Song L: Overexpression of astrocyte elevated gene-1 (AEG-1) is associated with esophageal squamous cell carcinoma (ESCC) progression and pathogenesis. Carcinogenesis 30: 894-901, 2009

16. Fan B, Wang YX, Yao T and Zhu YC: p38 mitogen-activated protein kinase mediates hypoxia-induced vascular endothelial growth factor release in human endothelial cells. Acta Physiol Sin 57: 13-20, 2005 .
17. Shen YM, Yang XC, Song ML, Qin $\mathrm{CH}$, Yang $\mathrm{C}$ and Sun $\mathrm{YH}$ : Growth inhibition induced by short hairpin RNA to silence survivin gene in human pancreatic cancer cells. Hepatobiliary Pancreat Dis Int 9: 69-77, 2010.

18. Vose JM: Peripheral T-cell non-Hodgkin's lymphoma. Hematol Oncol Clin North Am 22: 997-1005, 2008,

19. Fornari A, Piva R, Chiarle R, Novero D and Inghirami G: Anaplastic large cell lymphoma: one or more entities among T-cell lymphoma? Hematol Oncol 27: 161-170, 2009.

20. Windsor R, Stiller C and Webb D: Peripheral T-cell lymphoma in childhood: population-based experience in the United Kingdom over 20 years. Pediatr Blood Cancer 50: 784-787, 2008.

21. Kossakowska AE, Edwards DR, Prusinkiewicz C, Zhang MC, Guo D, Urbanski SJ, Grogan T, Marquez LA and Janowska-Wieczorek A: Interleukin-6 regulation of matrix metalloproteinase (MMP-2 and MMP-9) and tissue inhibitor of metalloproteinase (TIMP-1) expression in malignant nonHodgkin's lymphomas. Blood 94: 2080-2089, 1999.

22. Chow KU, Boehrer S, Geduldig K, Krapohl A, Hoelzer D, Mitrou PS and Weidmann E: In vitro induction of apoptosis of neoplastic cells in low-grade non-Hodgkin's lymphomas using combinations of established cytotoxic drugs with bendamustine. Haematologica 86: 485-493, 2001.

23. Sebti Y, Le Maux A, Gros F, De Guibert S, Pangault C, RouasFreiss N, Bernard M and Amiot L: Expression of functional soluble human leucocyte antigen-G molecules in lymphoproliferative disorders. Br J Haematol 138: 202-212, 2007.

24. Yang J, Wang S, Zhao G and Sun B: Effect of chemokine receptors CCR7 on disseminated behavior of human $\mathrm{T}$ cell lymphoma: clinical and experimental study. J Exp Clin Cancer Res 30: 51-59, 2011.

25. Sarkar D, Emdad L, Lee SG, Yoo BK, Su ZZ and Fisher PB: Astrocyte elevated gene-1: far more than just a gene regulated in astrocytes. Cancer Res 69: 8529-8535, 2009.

26. Chen W, Ke Z, Shi H, Yang S and Wang L: Overexpression of AEG-1 in renal cell carcinoma and its correlation with tumor nuclear grade and progression. Neoplasma 57: 522-529, 2010.

27. Song H, Li C, Li R and Geng J: Prognostic significance of AEG-1 expression in colorectal carcinoma. Int $\mathrm{J}$ Colorectal Dis 25: 1201-1209, 2010

28. Li C, Li R, Song H, Wang D, Feng T, Yu X, Zhao Y, Liu J, Yu X, Wang Y and Geng J: Significance of AEG-1 expression in correlation with VEGF, microvessel density and clinicopathological characteristics in triple-negative breast cancer. J Surg Oncol 103: 184-192, 2011.

29. Sun W, Fan YZ, Xi H, Lu XS, Ye C and Zhang JT: Astrocyte elevated gene-1 overexpression in human primary gallbladder carcinomas: An unfavorable and independent prognostic factor. Oncol Rep 26: 1133-1142, 2011.

30. Li C, Liu J, Lu R, Yu G, Wang X, Zhao Y, Song H, Lin P, Sun X, Yu X, Zhang Y, Ning X and Geng J: AEG-1 overexpression: a novel indicator for peritoneal dissemination and lymph node metastasis in epithelial ovarian cancers. Int J Gynecol Cancer 21: 602-608, 2011.

31. Song H, Li C, Lu R, Zhang Y and Geng J: Expression of astrocyte elevated gene-1: a novel marker of the pathogenesis, progression, and poor prognosis for endometrial cancer. Int J Gynecol Cancer 20: 1188-1196, 2010.

32. Yoo BK, Chen D, Su ZZ, Gredler R, Yoo J, Shah K, Fisher PB and Sarkar D: Molecular mechanism of chemoresistance by astrocyte elevated gene-1. Cancer Res 70: 3249-3258, 2010.

33. $\mathrm{Xu} \mathrm{JB}, \mathrm{Wu} \mathrm{H}, \mathrm{He}$ YL, Zhang $\mathrm{CH}$, Zhang LJ, Cai SR and Zhan WH: Astrocyte-elevated gene-1 overexpression is associated with poor prognosis in gastric cancer. Med Oncol 28: 455-462, 2011

34. Song L, Li W, Zhang H, Liao W, Dai T, Yu C, Ding X, Zhang L and Li J: Over-expression of AEG-1 significantly associates with tumour aggressiveness and poor prognosis in human non-small cell lung cancer. J Pathol 219: 317-326, 2009.

35. Khuda II, Koide N, Noman AS, Dagvadorj J, Tumurkhuu G, Naiki Y, Komatsu T, Yoshida T and Yokochi T: Astrocyte elevated gene-1 (AEG-1) is induced by lipopolysaccharide as toll-like receptor 4 (TLR4) ligand and regulates TLR4 signalling. Immunology 128: 700-706, 2009.

36. Emdad L, Lee SG, Su ZZ, Jeon HY, Boukerche H, Sarkar D and Fisher PB: Astrocyte elevated gene-1 (AEG-1) functions as an oncogene and regulates angiogenesis. Proc Natl Acad Sci USA 106: 21300-21305, 2009. 
37. Kehinde EO, Maghrebi MA and Anim JT: The importance of determining the aggressiveness of prostate cancer using serum and tissue molecular markers. Can J Urol 15: 3967-3974, 2008

38. Nardiello T, Jungbluth AA, Mei A, et al: MAGE-A inhibits apoptosis in proliferating myeloma cells through repression of Bax and maintenance of survivin. Clin Cancer Res 17: 43094319,2011

39. Jamieson NB, Carter CR, McKay CJ and Oien KA: Tissue biomarkers for prognosis in pancreatic ductal adenocarcinoma: a systematic review and meta-analysis. Clin Cancer Res 17: 3316-3331, 2011.

40. Liu L, Wu J, Ying Z, Chen B, Han A, Liang Y, Song L, Yuan J, $\mathrm{Li} \mathrm{J}$ and $\mathrm{Li} \mathrm{M}$ : Astrocyte elevated gene-1 upregulates matrix metalloproteinase-9 and induces human glioma invasion. Cancer Res 70: 3750-3759, 2010.
41. Wang F, Ke ZF, Sun SJ, Chen WF, Yang SC, Li SH, Mao XP and Wang LT: Oncogenic roles of astrocyte elevated gene-1 (AEG-1) in osteosarcoma progression and prognosis. Cancer Biol Ther 12: 539-548, 2011.

42. Nelson AR, Fingleton B, Rothenberg ML and Matrisian LM: Matrix metalloproteinases: biologic activity and clinical implications. J Clin Oncol 18: 1135-1149, 2000.

43. Peschos D, Damala C, Stefanou D, Tsanou E, Assimakopoulos D, Vougiouklakis T, Charalabopoulos K and Agnantis NJ: Expression of matrix metalloproteinase-9 (gelatinase B) in benign, premalignant and malignant laryngeal lesions. Histol Histopathol 21: 603-608, 2006. 